\title{
ARTICLE
}

\section{Prenatal inflammation exposure-programmed hypertension exhibits multi-generational inheritance via disrupting DNA methylome}

Xiao Guan ${ }^{1}$, Guo-rong Dan ${ }^{1}$, Yao Yang ${ }^{1}$, Yan $\mathrm{Ji}^{1}$, Wen-jing Lai ${ }^{1}$, Fang-jie Wang ${ }^{1}$, Meng Meng ${ }^{1}$, Bang-hui Mo ${ }^{1}$, Pei Huang ${ }^{1}$, Ting-ting You ${ }^{1}$, Ya-fei Deng ${ }^{1}$, Liang Song ${ }^{1}$, Wei Guo ${ }^{1}$, Ping $\mathrm{Yi}^{2}$, Jian-hua Yu ${ }^{3,4}$, Yuan Gao ${ }^{5}$, Wei-nian Shou ${ }^{1,6}$, Bing-bo Chen ${ }^{7}$, You-cai Deng ${ }^{1}$ and Xiao-hui Li ${ }^{1}$

The multi-generation heredity trait of hypertension in human has been reported, but the molecular mechanisms underlying multigenerational inheritance of hypertension remain obscure. Recent evidence shows that prenatal inflammatory exposure (PIE) results in increased incidence of cardiovascular diseases, including hypertension. In this study we investigated whether and how PIE contributed to multi-generational inheritance of hypertension in rats. PIE was induced in pregnant rats by intraperitoneal injection of LPS or Poly (I:C) either once on gestational day 10.5 (transient stimulation, T) or three times on gestational day $8.5,10.5$, and 12.5 (persistent stimulation, P). Male offspring was chosen to study the paternal inheritance. We showed that PIE, irrespectively induced by LPS or Poly $(I: C)$ stimulation during pregnancy, resulted in multi-generational inheritance of significantly increased blood pressure in rat descendants, and that prenatal LPS exposure led to vascular remodeling and vasoconstrictor dysfunction in both thoracic aorta and superior mesenteric artery of adult F2 offspring. Furthermore, we revealed that PIE resulted in global alteration of DNA methylome in thoracic aorta of F2 offspring. Specifically, PIE led to the DNA hypomethylation of G beta gamma (G $\beta \gamma$ ) signaling genes in both the F1 sperm and the F2 thoracic aorta, and activation of PI3K/Akt signaling was implicated in the pathologic changes and dysregulated vascular tone of aortic tissue in F2 LPS-P offspring. Our data demonstrate that PIE reprogrammed DNA methylome of cells from the germline/mature gametes contributes to the development of hypertension in F2 PIE offspring. This study broadens the current knowledge regarding the multi-generation effect of the cumulative early life environmental factors on the development of hypertension.

Keywords: hypertension; multi-generational inheritance; prenatal inflammation exposure; DNA methylation; G $\beta \gamma ;$ PI3K/Akt signaling; LPS; poly (I:C)

Acta Pharmacologica Sinica (2022) 43:1419-1429; https://doi.org/10.1038/s41401-021-00772-8

\section{INTRODUCTION}

Hypertension is the leading risk factor for cardiovascular diseases (CVDs), affecting the health of more than 1 billion people worldwide [1]. A recent multi-generation cohort study of Framingham Heart Study participants has demonstrated that early-onset hypertension not only in parents but also in grandparents could raise the risk for hypertension in their descendants, indicative of multi-generation heredity of hypertension [2]. However, the detailed mechanisms are largely unknown.

Prenatal inflammation exposure (PIE), a momentous event during pregnancy, could be caused by various pathogenesis, including "hot" inflammation after pathogens infections (virus, bacterial, and parasites infection) and also "cold" inflammation in several chronic diseases (such as obesity, maternal diabetes or hyperglycaemia, hypertension, pre-eclampsia, and smoking) [3]. Decades of research works have shown the close association between PIE and neuropsychiatric disorders, such as autism and schizophrenia [4]. Recent epidemiological and experimental studies have also revealed that prenatal influenza or bacterial exposure results in increased incidence of CVDs, including hypertension (summarized in our and others' recent published reviews) $[3,5-7]$, which provide a close linkage between PIE and CVDs in adult offspring (called as PIE-programed CVDs). Due to the pandemic, over 150 million people have Coronavirus disease (COVID-19). Pregnant women with COVID-19 also showed an obvious proinflamatory status, such as increased levels of IL-6,

\footnotetext{
${ }^{1}$ Institute of Materia Medica, College of Pharmacy, Army Medical University (Third Military Medical University), Chongqing 400038, China; ${ }^{2}$ Department of Obstetrics and Gynecology, The Third Affiliated Hospital of Chongqing Medical University, Chongqing 401120, China; ${ }^{3}$ The Ohio State University Comprehensive Cancer Center and the James Cancer Hospital, Columbus, OH 43210, USA; ${ }^{4}$ Department of Hematology \& Hematopoietic Cell Transplantation, City of Hope National Medical Center and Beckman Research Institute, Duarte, CA 91010, USA; ${ }^{5}$ Southwest Hospital/Southwest Eye Hospital, Army Medical University (Third Military Medical University), Chongqing 400038, China; ${ }^{6}$ Herman B Wells Center for Pediatric Research, Department of Pediatrics, Indiana University School of Medicine, Indianapolis, IN 46202, USA and ${ }^{7}$ Laboratory Animal Center, Army Medical University (Third Military Medical University), Chongqing 400038, China
}

Correspondence: Bing-bo Chen (chenbb81@126.com) or You-cai Deng (youcai.deng@tmmu.edu.cn) or Xiao-hui Li (Ipsh008@aliyun.com)

These authors contributed equally: Xiao Guan, Guo-rong Dan, Yao Yang, Yan Ji

Received: 31 January 2021 Accepted: 1 September 2021

Published online: 30 September 2021 
IFNy, C-reactive protein, and D-dimer, in periphery blood serum [8]. All these evidence demonstrate a great need to elucidate the detailed mechanisms for PIE-induced CVDs, which may provide new insight into preventing the continuous increasing rate of CVDs incidence post COVID-19 pandemic [9].

Rats have been used as the primary model for studying the genetic mechanisms of hypertension [10]. A rat model of PIEprogramed CVDs has been used previously by treating pregnant Sprague-Dawley (SD) rats with lipopolysaccharide (LPS, the main component of gram-negative bacteria cellular wall) and polyriboinosinic-polyribocytidylic acid (Poly (I:C)), a substitute for viral dsRNA and a ligand of toll-like receptor 3), during the secondary semester, a similar strategy that is extensively used in studying the mechanisms of PIE-induced neuropsychiatric disorders [4]. Irrespective of transient stimulus (one time on gestational day 10.5) or persistent stimulus (three times on gestational day 8.5, 10.5, and 12.5) of LPS or Poly (I:C) challenge, the F1 offspring showed aortic dysfunction and eventually developed hypertension at elder age [11-13]. However, whether PIE could contribute to the multigeneration heredity of hypertension remains largely unknown.

Recent studies have demonstrated that epigenomic features, such as DNA methylation by covalent modifications, can be transmitted for several generations. DNA methylation pattern could better reflect the cumulative effects of environmental and lifestyle factors than expression profiles that are subject to transient changes [14-16]. To this end, in this current study, we investigated the phenotype of transgenerational inheritance in multiple generations relevant to PIE-programmed hypertension and explored the potential mechanisms regarding DNA methylation. We found that PIE-programed hypertension, irrespective of induced by prenatal LPS or Poly $(\mathrm{I}: \mathrm{C})$ stimulation and irrespective of stimulation duration, was inherited by several generations, accompanied by altered DNA methylome. Specifically, PIE led to the DNA hypomethylation of $G$ beta gamma $(G \beta \gamma)$ signaling genes in both the sperm of F1 offspring and the thoracic aorta of the second generation (F2) offspring, accounting for the pathologic changes and dysregulated vascular tone of aortic tissue via phosphatidylinositol 3-kinase/protein kinase B (PI3K/Akt) signaling pathway. These data demonstrated that PIE reprogrammed the DNA methylome of cells from the germline/mature gametes, which could be inherited by several generations, contributing to the increased risk of hypertension.

\section{MATERIALS AND METHODS}

\section{Experimental animals}

Nonparous SD rats were purchased from the Animal Centre of Army Medical University (Chongqing, China). All rats were bred and housed in pathogen-free conditions, at $22-25^{\circ} \mathrm{C}$, periodic air changes, and free access to water and food, and had 12-h light/ dark cycle, at the Experimental Animal Center of the Animal Centre of Army Medical University (Chongqing, China), according to ARRIVE guidelines and the guidelines of Institutional Animal Care and Use Committee. All experiments performed were approved by the Institutional Animal Care and Use Committee at the Animal Centre of Army Medical University (Chongqing, China), and complied with the NIH guidelines or the guidelines from Directive 2010/63/EU of the European Parliament on the protection of animals used for scientific purposes.

\section{Experimental design}

F0 maternal inflammatory stimulation: time-dated nulliparous pregnant SD rats $(250-300 \mathrm{~g})$ were randomly divided into five groups ( $n=8$ for each group), intraperitoneally (i.p.) injected with saline on embryonic day 8.5, 10.5, and 12.5 (E8.5, 10.5, and 12.5); LPS $(0.79 \mathrm{mg} / \mathrm{kg}$, Sigma-Aldrich, L2630, St. Louis, MO, USA) on E8.5, 10.5, and 12.5; LPS $(0.79 \mathrm{mg} / \mathrm{kg})$ on E10.5; Poly (l:C) $(4 \mathrm{mg} / \mathrm{kg}$, SigmaAldrich, P1530) (only included the weight of the Poly (I:C) itself on
E8.5, 10.5, and 12.5); and Poly (I:C) (4 mg/kg) (only included the weight of the Poly (I:C) itself on E10.5, named as Control, LPS-P, LPS-T, Poly ( $(: C)-P$, and Poly ( $(: C)-T$ group, respectively). " $P$ " indicates persistent stimulation, and " $\mathrm{T}$ " indicates transient stimulation, respectively [12]. For LPS-T and Poly (I:C)-T groups, pregnant SD rats were also i.p. injected with saline on E8.5 and E12.5. No convulsions, deaths, or miscarriage occurred after LPS or Poly (I:C) injection in all the treated pregnant rats. Pups were delivered around 21 days of pregnancy and no obvious deformities were found.

After delivery, litter size was adjusted to eight pups per dam by random selection to avoid the disproportion of nutrient availability during lactation. Offspring rats from F0 mothers were designated as F1 control or PIE offspring. Multi-generational inherited rat model of PIE referred to the previously reported mouse model of intrauterine growth restriction [17]. F1 pups were nursed freely and weaned at the age of 4 weeks and provided with standard chow ad libitum. As it is difficult to rule out the influence of intrauterine microenvironment changes, maternal metabolism, and behavior on the offspring physiology by using maternal transmission model to clarify transgenerational inheritance [18], we chose male offspring to study the paternal inheritance. External control virgin females (250-300 g) were bred with either F1 control or PIE males, randomized selected from each litter of rats, to generate F2 PIE descendants, named as F2 control, F2 LPS-P, F2 LPS-T, F2 Poly (I:C)-P, F2 Poly (I:C)-T, respectively (Fig. 1a). At birth, F2 litters were adjusted to eight pups per dam and had standard chow ad libitum at weaning at the age of 4 weeks. F2 control and F2 LPS-P males were bred with external control females to generate F3 offspring, while F3 LPS-P males were further bred with external control females to generate F4 offspring (Fig. 1a). For F1-F4 offspring, pregnant rats and pups were no longer subjected to the stimulation with LPS or Poly (I:C). Rats were anesthesized with sodium pentobarbital $(240 \mathrm{mg} / \mathrm{kg}$, i. p.) and were sacrificed by cervical dislocation if necessary.

Blood pressure measurement

Systolic blood pressure (SBP) was measured by the tail-cuff system (Softron BP-2010A, Softron Biotechnology Co., Beijing, China) every 4 weeks starting at 8 and ending at the age of 28 weeks, as described previously [12]. The investigators were blinded for measuring the blood pressure.

\section{Histological analysis}

The rats were anesthetized via intra-abdominal injection with pentobarbital $(100 \mathrm{mg} / \mathrm{kg}$, Sigma-Aldrich, P-010) and euthanized by cervical dissociation. The thoracic aortas and superior mesenteric arteries were fixed in 4\% paraformaldehyde (Sigma-Aldrich, 158127) for 7 days, soaked in 30\% sucrose, embedded in paraffin wax, sectioned $(4 \mu \mathrm{m})$, and then stained with haematoxylin and eosin as described previously [19]. The sections were visualized under an Olympus BH-2 microscope. The values of vascular wall thickness, cross-sectional area, and vascular smooth muscle cells (VSMCs) width were quantified by using ZEN 2.3 (blue edition). The investigators were blinded for acquiring and quantifying the images.

\section{Immunofluorescence staining}

The protein levels of eNOS in thoracic aortas were determined by immunofluorescence staining, as described previously [19]. Goat anti-mouse IgG H\&L (Alexa Fluor ${ }^{\circledR}$ 647) (ab150115) antibodies (Abcam, La Jolla, CA, USA) were used for an additional $1 \mathrm{~h}$ incubation after anti-eNOS (ab76198) antibodies (Abcam, La Jolla, (A, USA) incubation and washing. Sections were then incubated with $1 \mathrm{mg} / \mathrm{mL}$ DAPI for $10 \mathrm{~min}$, washed three times with PBS, and mounted into Vectashield ${ }^{\oplus}$ mounting medium (Vector Laboratories, Burlingame, CA, USA). The coverslips were visualized under a Leica confocal laser scanning microscope (Leica, Wetzlar, Germany). The investigators were blinded for acquiring the images. 
Vascular reactivity assessment

The procedure of vascular reactivity assessment was performed as described previously [20]. Briefly, rats were euthanized, thoracic aortas and superior mesenteric arteries were cut into 4-mm rings, stored in an oxygenated Krebs-Henseleit $(\mathrm{K}-\mathrm{H})$ solution $(\mathrm{mM}$ : $\mathrm{NaCl}, 11.8 ; \mathrm{KCl}, 0.47 ; \mathrm{KH}_{2} \mathrm{PO}_{4}, 0.13 ; \mathrm{MgSO}_{4}, 0.045 ; \mathrm{NaHCO}_{3}, 2.5 ;$ $\mathrm{CaCl}_{2}, 2$; glucose, 11.1$)$ at $4{ }^{\circ} \mathrm{C}$ and gassed with a carbogenic mixture $\left(95 \% \mathrm{O}_{2}\right.$ and $\left.5 \% \mathrm{CO}_{2}\right)$. Vascular rings were suspended on triangular-shaped stainless steel hooks in warmed $\left(37^{\circ} \mathrm{C}\right)$ and aerated $\left(95 \% \mathrm{O}_{2}, 5 \% \mathrm{CO}_{2}\right) \mathrm{K}-\mathrm{H}$ solution using Automatic organ bath (Panlab, Harvard Apparatus, USA). A computerized force transducer (Powerlab System, AD Instruments, Australia) was used to record the isometric tension of the aortic and mesenteric arteries rings. After $60 \mathrm{~min}$ of equilibration, each ring was challenged with $\mathrm{KCl}(60 \mathrm{mM})$. Phenylephrine (PE) (Sigma-Aldrich, $\mathrm{P} 1240000)$ was added cumulatively $\left(10^{-10}-3 \times 10^{-6} \mathrm{M}\right)$ to the bath to establish a concentration-response contraction curve, and the contraction was expressed as a percentage of the contraction produced by $60 \mathrm{mM} \mathrm{KCl}$ for each ring. Acetylcholine (ACh) (SigmaAldrich, A6625) or sodium nitroprusside (SNP) (Sigma-Aldrich, $1614501)$ with cumulative concentrations $\left(10^{-10}-3 \times 10^{-6} \mathrm{M}\right)$ were added to endothelium-intact or de-endothelialized aortic rings, respectively, to establish a concentration-response relaxation curve. Relaxation response was expressed as a percentage of the maximal contractile response induced by $P E\left(3 \times 10^{-6} \mathrm{M}\right)$. Concentration-response curves were analyzed by linear regression analysis (fitted to the Hill equation) using Prism (GraphPad Software, CA, USA), and expressed as the values of $\mathrm{pD}_{2}\left(-\log \mathrm{EC}_{50}\right)$. To determine the role of PI3K-Akt in vascular function, PI3K inhibitor (LY294002, $20 \mu \mathrm{M}$ ) (Sigma-Aldrich, 440202) [21] was added 30 min before vascular reactivity assessment and stayed in the Automatic organ bath during the whole vascular reactivity assessment.

\section{MeDIP-seq}

Four samples from both the F2 control and F2 LPS-P groups were used for methylated DNA immunoprecipitation sequencing (MeDIP-seq). Sample preparation and MeDIP-Seq were accomplished by Kangcheng Bio-tech, Shanghai, China. Briefly, highquality genomic DNA (gDNA) isolated from thoracic aorta tissues (3.5 - $4.5 \mathrm{mg}$ ) was fragmented to a size range of 200-700 bp with a Diagenode Bioruptor, and a portion of the denatured DNA fragments was taken as input DNA. About $1 \mu \mathrm{g}$ fragmented DNA was prepared for Illumina HiSeq 2000 sequencing by using the following steps: (1) end repair of DNA samples with T4 DNA polymerase, Klenow DNA polymerase, and T4 polynucleotide kinase (T4 PNK); (2) a single " $A$ " base was added to the $3^{\prime}$ ends with Klenow (exo minus) polymerase; (3) ligation of Illumina's genomic adapters to DNA fragments; (4) MeDIP to the enrich methylated DNA by using anti-5-methylcytosine antibodies (Abcama, ab10805); (5) PCR amplification of the enriched precipitated DNA fragments; and (6) gel purification to extract the DNA fragments with length of $300-800 \mathrm{bp}$. The completed libraries were quantified by Agilent 2100 Bioanalyzer. The libraries were then denatured with $0.1 \mathrm{M} \mathrm{NaOH}$ (Sigma-Aldrich, S8045) to generate single-stranded DNA molecules, captured on Illumina flow cell and amplified in situ. The libraries were then sequenced on the Illumina HiSeq 2000 following the TruSeq SBS Kit v5 protocol.

Raw files were processed by FastQC for quality control. Quality and adapter trimming was performed using Cutadapter. Reads were mapped to the respective reference genomes ( $(n 6)$ using BOWTIE software (V2.1.0). The average alignment rate of MeDIPseq reads from all samples was $96.83 \%$, and on average $76.61 \%$ of reads were uniquely mapped to the reference genome (Supplementary Fig. S1a). Average enrichment score was 1.25 in all tested samples (Supplementary Fig. S1b). MEDIPs saturation analysis indicated that Pearson's correlation values of all samples were close to one, and the read coverage was sufficient for next downstream analysis (Supplementary Fig. S1c). The CpG coverage plots also illustrated similar shape between the two groups and indicated an effective MeDIP enrichment. On average, $12.45 \%$ of mapped reads did not cover a pattern (Supplementary Fig. S1d). The data showed that methylated DNAs were properly captured by MeDIP-seq used in this study. Differentially methylated regions (DMRs) were identified using MEDIPS package version 1.12.0 with the condition that log 2 (fold-change) $\geq 2$ and FDR $<0.05$. Then we used ChIPSeeker to annotate DMRs using rn6 known gene table from UCSC. The reads were visualized and individual genes were examined using WashU Epigenome Browser.

To conduct enrichment analysis, the package cluster Profiler (version 3.2.14) of $\mathrm{R}$ (version 3.3.3) was used for analyzing the Kyoto Encyclopedia of Genes and Genomes (KEGG) pathways and Gene Ontology (GO) processes based on the DMR associated genes. The Ingenuity ${ }^{\oplus}$ Pathway Analysis (IPA) (https://www.qiagen. com/us/products/informatics-and-data/interpretation-content-

databases/ingenuity-pathway-analysis/\#orderinginformation) was also used for analyzing the canonical pathways and interaction networks. $P$ value for the pathway enrichment is calculated by using Fisher's exact test, and $P<0.05$ is considered statistically significant. Then the results were imported to Cytoscape (version 3.4.0) to construct gene-pathway network.

\section{BSP}

gDNA isolated from both F2 thoracic aortas and F1 sperm was carried out by using QIAamp DNA Mini Kit (QIAGEN, 51304, Hilden, Germany). Bisulfate treatment of gDNA was performed by using EZ DNA Methylation-Gold $\mathrm{Kit}^{\mathrm{TM}}$ (ZYMO Research, D5005, Irvine, (A, USA), according to the manufacturer's instructions. PCR primers were designed with MethPrimer (http://www.urogene. org/methprimer/), and bisulfite-treated DNA was amplified with EpiTaq HS polymerase (Takara, R110Q, Beijing, China).

The PCR products were separated by using agarose gel electrophoresis and purified by using the Wizard ${ }^{\oplus}$ SV Gel and PCR Clean-Up System (Promega, A9281, Madison, WI, USA). Purified DNA fragments were cloned into pMD T-19 (Takara, No. 6013Code) and ten clones from each PCR assay were subjected to next direct sequencing. The final sequence results were analyzed by online QUMA (quantification tool for methylation analysis) software [22]. Primer sets used in bisulfate modified PCR are shown in Supplementary Table S1.

\section{Real-time RT-PCR}

Total RNA was extracted from thoracic aortas by using TRIzol Reagent method (Invitrogen, 15596-026) and then reversetranscribed into cDNA by using a Bestar quantitative real-time polymerase chain reaction (qPCR) RT Kit (DBI Bioscience, DBI-2220, Ludwigshafen, Germany), as described previously [23]. qPCR was performed with Bestar Sybr Green qPCR Master Mix (DBI Bioscience, DBI-2043) according to the following conditions: 2 min at $95^{\circ} \mathrm{C} ; 40$ cycles at $95^{\circ} \mathrm{C}$ for $10 \mathrm{~s}, 60^{\circ} \mathrm{C}$ for $12 \mathrm{~s}, 68^{\circ} \mathrm{C}$ for $10 \mathrm{~s}$, $72{ }^{\circ} \mathrm{C}$ for $20 \mathrm{~s} ; 5 \mathrm{~min}$ at $72{ }^{\circ} \mathrm{C}$, using $\mathrm{ABI}$ Prism 7700 sequence detection system (Applied Biosystems). Primer sequences for qPCR were designed by Pubmed Primer-BLAST and listed in Supplementary Table S2. Relative mRNA expression was calculated by normalizing the circulating threshold with $\beta$-actin and then normalizing the relative gene expression to the control group.

\section{Immunoblotting}

Protein expression in thoracic aortas was determined by immunoblotting, as described previously [23]. Briefly, thoracic aortas were homogenized with T-PER tissue protein extraction reagent (Thermo-Pierce, 78510, Rockford, IL, USA) containing protease inhibitor cocktails (Sigma-Aldrich, P8340) and protein concentrations were determined by BCA Protein Assay Kit (BioRad, 5000001, Hercules, CA, USA). Proteins from each sample (20 
$\mu \mathrm{g})$ were separated by $8 \%-12 \%$ Tris SDS-PAGE gel electrophoresis and transferred to polyvinylidene difluoride membranes (Bio-Rad Laboratories, Inc., 1620256, CA, USA). After blocking with 5\% nonfat dry milk, membranes were incubated with anti-p-Akt (Ser473), anti-p-S6 Ribosomal Protein (Ser235/236), anti-Akt (C73H10), antiS6 Ribosomal Protein (Cell Signaling Technology, 4058; 4856; 2938; 2217, Beverly, MA, USA), or anti- $\beta$-actin (AC-15) (SigmaAldrich, A5441) antibodies overnight at $4{ }^{\circ} \mathrm{C}$, and incubated with the secondary antibodies (Santa Cruz, sc-2004; sc-2005, Santa Cruz, CA, USA) at room temperature for $1 \mathrm{~h}$. Proteins were visualized with enhanced chemiluminescence reagents (ThermoPierce, Rockford, IL, USA) and the blots were exposed to hyper film. Results were quantified by using Quantity-one software (BioRad).

\section{Statistical analysis}

Results are expressed as mean \pm SEM. Statistical analysis was performed by using an unpaired Student's $t$ test (two unpaired groups) or two-way ANOVA followed by Sidak's multiple comparison test (multiple groups comparison) by using SPSS 18.0 software (SPSS Inc., Chicago, IL, USA). All of the statistical tests were two-tailed, and $P<0.05$ was considered to represent a significant difference.

\section{RESULTS}

PIE-programmed hypertension is inherited by multi-generation in rat offspring

The Bisulfite sequencing PCR (BSP) of F1 PIE male offspring, including F2 LPS-P, F2 LPS-T, F2 Poly (I:C)-P, F2 Poly (I:C)-T, was significantly higher than that in $\mathrm{F} 1$ control offspring (Supplementary Fig. S2a). To interrogate whether the increased blood pressure could be inherited by multiple generations, we first determine the SBP of F2 PIE offspring. The SBP of F2 PIE offspring from whose fathers experiencing transient or persistent treatment with LPS or Ploy (I:C) prenatally was all significantly elevated from 8 weeks old onwards than that of F2 control offspring (Fig. 1a-c), which is similar to those found in F1 PIE progenies $[12,13]$. Compared with F2 control offspring, more F2 PIE offspring were distributed at higher blood pressure values, while the maximum value of blood pressure in F2 PIE offspring was also greater than that of control F2 offspring, irrespective of F1 fathers experiencing prenatal LPS or Poly (I:C) exposure (Fig. 1d, e). Further analysis revealed that the SBP of both male and female F2 PIE offspring showed statistical difference with F2 control offspring, indicative of no sexual difference (Supplementary Fig. S2b-e). We further selected F2 LPS$P$ model to verify the transgenerational inheritance of increased SBP in F3 and F4 offspring. The SBP in both F3 and F4 offspring from $F 1$ fathers experiencing prenatal persistent treatment with LPS (F3 LPS-P and F4 LPS-P) showed similar increased levels as seen in F2 LPS-P offspring (Fig. 1f-i).

Histological image of vessel wall from F2 control thoracic aorta clearly displayed the tunica intima-media-adventitia layering, and the media consist of regularly organized musculo-elastic complexes. However, the morphologic observation revealed that F2 LPS-P offspring (whose fathers experiencing three times of LPS stimulation on E8.5, 10.5, and 12.5 prenatally) showed obvious vascular remodeling in both thoracic aortas and mesenteric arteries, such as increased arterial vascular wall thickness, wall thickness/lumen diameter ratio, and VSMCs width (Fig. 2a, b). Some of the endothelial cells of thoracic aortas from F2 LPS-P offspring were seen to be necrotic and detached from the subendothelial layer. Fragmented and unclear internal elastic fibers were also exhibited in thoracic aorta of F2 LPS-P offspring (Fig. 2a, b). In addition, hypertrophic and disarranged VSMCs were observed migrating and breaking through the internal elastic lamina (Fig. 2a, b). In addition, we also determined the changes of endothelial structure by immunofluorescence staining of eNOS, which is a critical marker for endothelium integrity in the thoracic aorta of both F2 control and F2 LPS-P offspring. The fluorescently labeled eNOS in the endothelium are shown in red as indicated by the arrow. The fluorescent labeling of eNOS in F2 control showed smooth and intact vascular endothelium, while the endothelium was shrunken, broken, and had increased gaps in F2 LPS-P offspring. This result suggests that the endothelium of thoracic aorta in F2 LPS-P offspring has obvious tissue structure abnormalities (Supplementary Fig. S3a).

The vascular reactivity assay also revealed that both the thoracic aorta and mesenteric artery from F2 LPS-P offspring showed dramatically higher contractile responses to $P E$ without affecting the ACh-induced vasodilation (Fig. 3a-d). As renin-angiotensin system (RAS) is critical to vascular contraction [1, 18], we next determined the mRNA levels of genes in RAS family, such as angiotensin-converting enzyme (ACE), angiotensin II receptor type 1 (AT1R), angiotensinogen (Agt) in both the thoracic aorta and SMA of both F2 control and F2 LPS-P offspring. The data revealed that in the thoracic aorta and SMA of F2 LPS-P, the mRNA expression of $A C E$, which is responsible for catalyzing the conversion of angiotensin I to angiotensin II, was significantly higher than F2 control offspring (Supplementary Fig. S3b).

Therefore, our data revealed that PIE induced multigenerational effect specifically on hypertension.

PIE alters overall methylation patterns in the aortic tissue of F2 offspring

Epigenetic mechanisms, referring to chromatin-based mechanisms without affecting DNA sequence, have emerged as a major goal in researching on hypertension, including DNA methylation, histone modification, microRNAs, and long noncoding RNAs [24]. DNA methylation pattern could better reflect the cumulative effects of environmental and lifestyle factors than expression profiles that are subject to transient challenges $[16,25]$. It is also implicated in the transmission of environmentally acquired phenotypes [26]. Therefore, we next determined the DNA methylome distribution of the thoracic aorta from F2 PIE offspring by MeDIP-seq, and followed by the verification in PIE F2 sperm samples. To produce the most robust phenotype, PIE F2 LPS-P offsprings at the age of 16 weeks from whose fathers experiencing prenatal persistent LPS stimulation were chosen. Our first set of data demonstrated 1111 hypomethylated DMRs and 1696 hypermethylated DMRs (loci with a mehylation change $>1.5$-fold and an edge $P$ value $<1 \times 10^{-4}$; Supplementary Fig. S4a, b and Supplementary Data 1 and 2) in thoracic aortas between F2 control and F2 LPS-P offspring, indicative of a global changed DNA methylation occurring in PIE F2 offspring. BSP validation of three hypomethylated (Cfhr1, Ndrg2, and Cox7a2l) and three hypermethylated (Fgfr2, Pfdn1, and Srpk2) DMRs within the promoter region or with the greatest fold-change between F2 control and F2 LPS-P offspring was shown to be remarkably consistent with the findings derived from MeDIP-seq data (Supplementary Fig. S5).

Annotation with genomic features illustrated that the majority of both hypomethylated and hypermethylated DMRs were located in intron, extron, 5'UTR, and $3^{\prime}$ UTR in intragenic elementassociated regions. Interestingly, hypomethylated DMRs were distributed more at the proximal promotor region of $-1 \mathrm{~kb}$ upstream and $+500 \mathrm{bp}$ downstream of the transcriptional start site (TSS) (labeled as Promoter-TSS) and the region of the transcriptional termination site than those in hypermethylated DMRs (Supplementary Fig. S4c). David GO Biological Process enrichment and KEGG analysis demonstrated that genes with hypomethylated DMRs were mainly enriched in positive regulation of RNA transcription and gene expression, endothelial cell migration, NF-kB transcription factor activity, MAPK activation, Ras, and Jak-STAT signaling pathway (Fig. 4a, b), all of which were implicated in the cardiovascular pathological changes and 

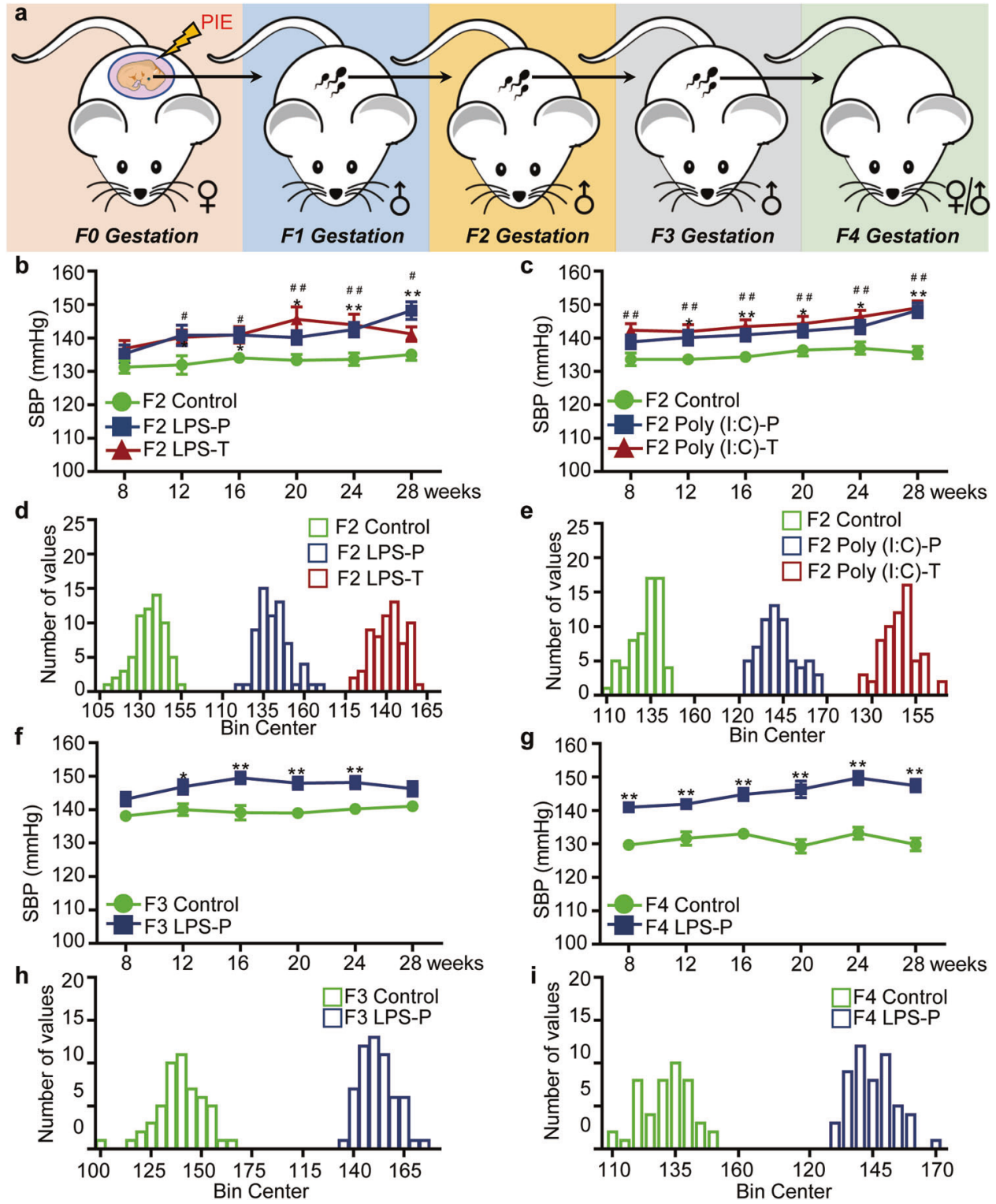

Fig. 1 Elevated systolic blood pressure (SBP) in adult F2-F4 descendants from F1 fathers experiencing prenatal inflammation exposure (PIE). a Schematic diagram for experimental design. F1 generation: dams were randomized on embryonic day (E) 8.5 to control, LPS-P, LPS-T, Poly (l:C)-P, or Poly (l:C)-T groups. Dams of LPS-P or Poly (l:C)-P were intraperitoneally injected with LPS (0.79 mg/kg) or Poly (l:C) potassium salt $(4 \mathrm{mg} / \mathrm{kg}$ based on the weight of Poly (I:C) itself) on E8.5, 10.5 and 12.5 (P indicates persistent), while dams of LPS-T or Poly (I:C)-T were intraperitoneally injected with LPS or Poly $(\mathrm{I}: \mathrm{C})$ on E10.5 (T indicates transient). F2-F4 generation: external control virgin females were mated with F1-F3 male offspring from each group to produce: F2 or F3 or F4 control, LPS-P, LPS-T, Poly (I:C)-P, or Poly (I:C)-T offspring, respectively. Postnatal litters were equalized to eight pubs and fed ad libitum for each generation. b, c The SBP of F2 offspring from the age of 8-28 weeks by tail-cuff method (b and $\mathbf{c})$ for whose paternal line experiencing prenatal LPS and Poly $(\mathrm{I}: \mathrm{C})$ stimulation, respectively. Data are expressed as mean \pm SEM. ${ }^{*} P<0.05$ and ${ }^{* *} P<0.01$ denote the statistical comparison between control and F2 LPS-P (b) or F2 Poly (l:C)-P (c) groups at the same time point, while ${ }^{\#} P<0.05$ and ${ }^{\# \#} P<0.01$ denote the statistical comparison between control and F2 LPS-T (B) or F2 Poly (l:C)-T (c) group at the same time point (unpaired Student's $t$ test for the same time point). d, e A histogram representing the distribution of SBP frequency in F2 offspring for each group at 16 weeks is shown (d and e for whose paternal line experiencing prenatal LPS and Poly (I:C) exposure, respectively). $\mathbf{f}, \mathbf{g}$ The SBP in F3 (f) and F4 (g) descendants from F1 control and LPS-P offspring. $\mathbf{h}, \mathbf{i}$ The distribution of SBP frequency in F3 (h) and F4 (i) descendants from F1 control and LPS-P offspring. Data are expressed as mean \pm SEM. ${ }^{*} P<0.05$ and ${ }^{*} P<0.01$, versus the same generation control offspring at the same time point (f-g, unpaired Student's $t$ test for the same time point). The numbers of animals used in each group were eight from eight different litters.

activation of vascular remodeling signaling pathways $[27,28]$. In addition, genes with hypermethylated DMRs were mainly enriched in brain and nervous system development, synapse assembly, CAMP, and TGF- $\beta$ signaling pathway (Fig. 4c, d). These suggested that genes with hypomethylated DMRs were mainly involved in the development of multi-generational inherited hypertension in PIE descendants, which was consistent with the findings in previous human epigenetic contribution to hypertension $[29,30]$. This is also consistent with recent studies that heritable hypomethylated chromosomal segments could be propagated for eight or more generations [31].

IPA software package to identify the enriched canonical pathways and IPA interaction networks demonstrated that there were several significantly enriched signaling pathways in genes 
a

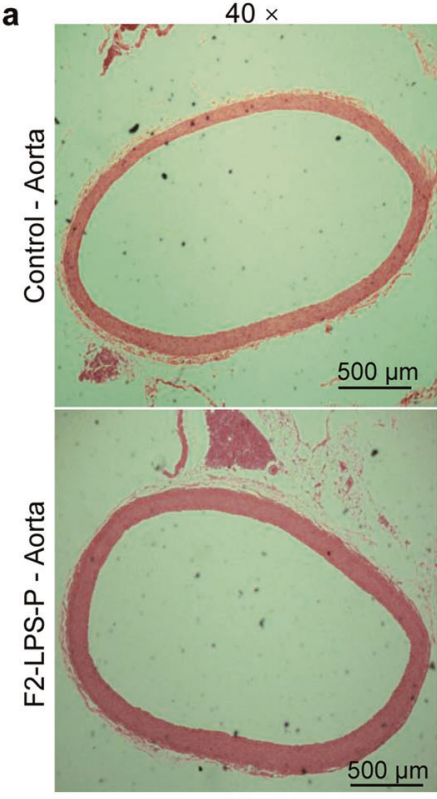

$40 \times$

$b$
के
$\frac{1}{0}$
응
0

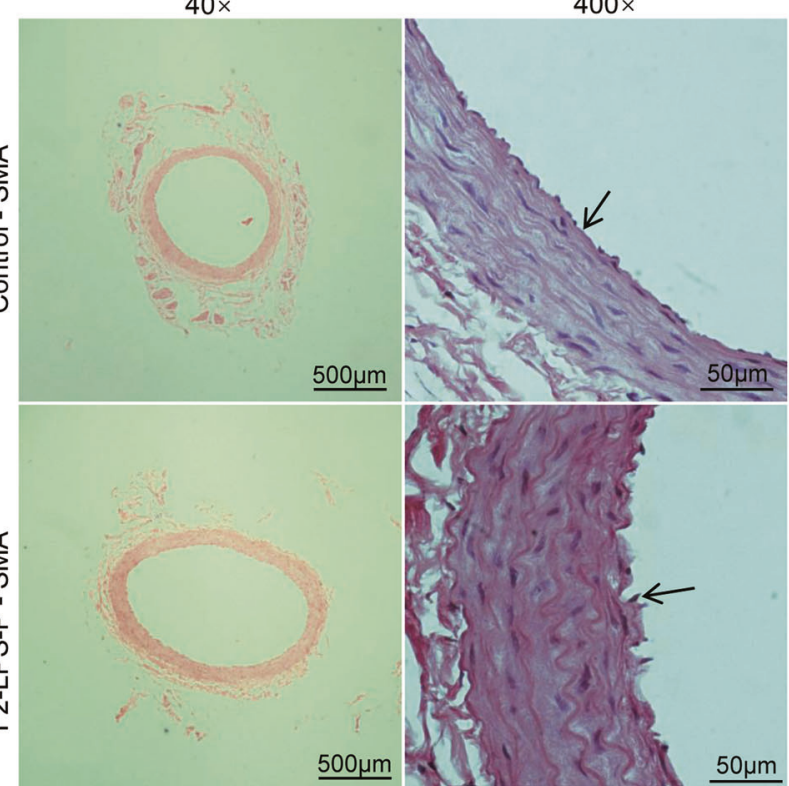

$400 \times$

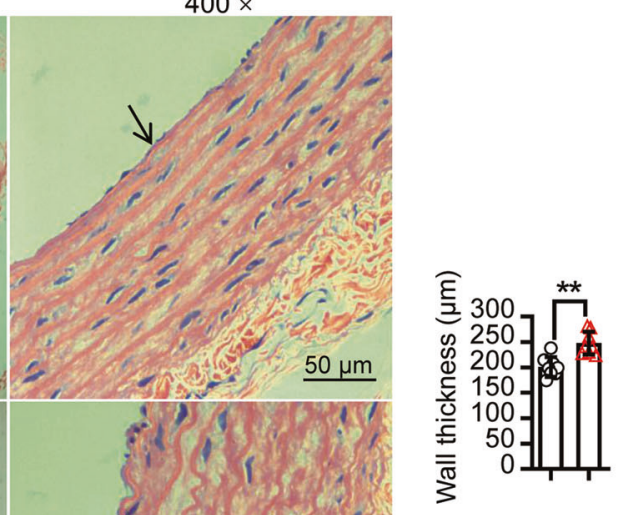

$\square$ F2 Control
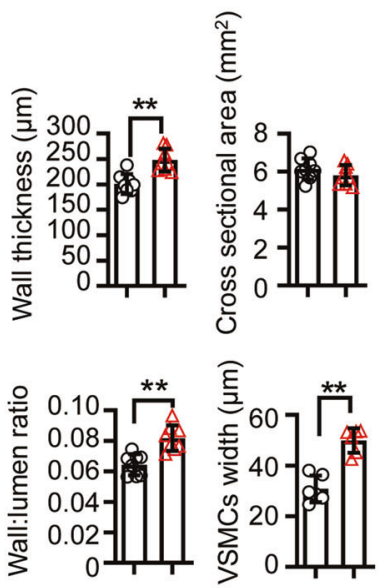
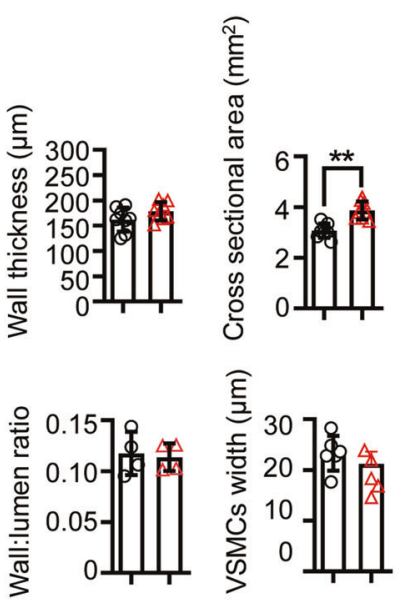

Fig. 2 Prenatal LPS exposure leads to vascular remodeling in both the thoracic aorta and superior mesenteric artery (SMA) of adult F2 offspring. a, b Histology of thoracic aortas (a) and SMA (b) from 16-week old F2 offspring by haematoxylin-eosin (H\&E) staining. Representative microphotographs with different magnification for each group are shown. Vessel wall nearby the arrow direction represents endothelium. The values of vascular wall thickness, cross-sectional area, wall:lumen ratio, and VSMC width were quantified by using NISElements BR software. Data are presented as means \pm SEM. $n=6$ from 3 litters for each group. Each dot represents one rat. ${ }^{* *} P<0.01$ denotes the statistical comparison between two marked groups (unpaired Student's $t$ test).

with hypomethylated DMRs associated with vascular remodeling and the pathogenesis of hypertension, such as GBY signaling, JAK/ STAT signaling, renin-angiotensin signaling, LPS-stimulated MAPK signaling, p53 signaling, p70S6K signaling, GPCR signaling, and ERK/MAPK signaling pathways (Fig. $4 \mathrm{f}$ and Supplementary Data 3) $[27,28,32]$. GßY signaling is the fourth most significant canonical pathway enriched in genes with hypomethylated DMRs $\left(P=4.81 \times 10^{-4}\right.$, overlap $\left.=12.5 \%\right) \quad($ Fig. $4 \mathrm{e})$. The IPA network interaction analysis suggested that $G \beta \gamma$ signaling was closely correlated with GPCR, JAK/STAT, LPS-stimulated MAPK, and ERK/ MAPK signaling (at least four genes shared by both signaling pathways) (Fig. 4f). This suggested that gene clusters in $G \beta \gamma$ signaling with hypomethylated DMRs might play a core role in the vascular pathological changes and development of hypertension in PIE F2 offspring.

Validation of hypomethylated DMRs of genes clustered in $G \beta Y$ signaling in both the F2 thoracic aorta and F1 sperm of PIE LPS-P offspring

As stated above, we next validated the mRNA expression levels and DNA methylation status within the promoter region of the genes in GBY signaling pathways by $\mathrm{QPCR}$ and BSP, respectively. The data demonstrated that the mRNA expression of the genes in G $\beta$ Y signaling showed an increased trend in thoracic aortas of PIE F2 LPS-P offspring. Among these genes, the mRNA expression levels of Sos1, Sos2, Gng10, Prkacb, and Pik3c3 showed statistical 
a

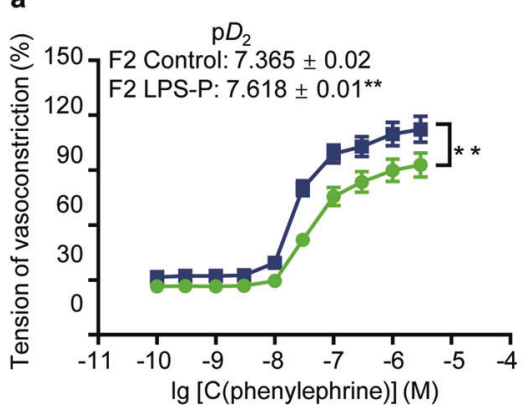

C

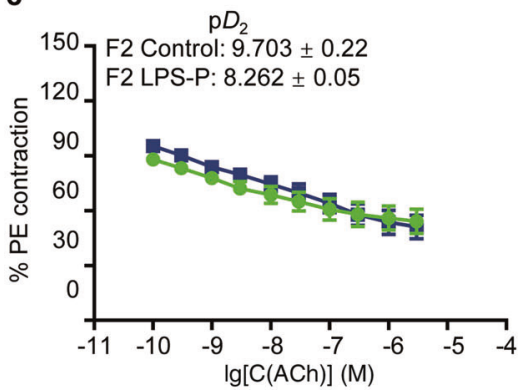

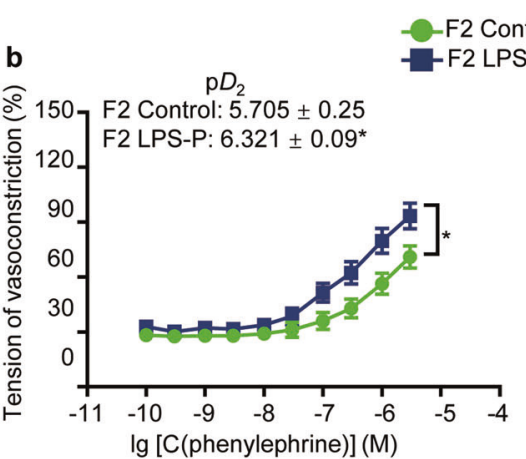

d

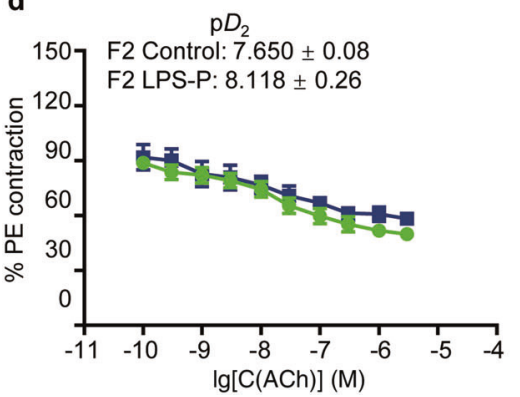

Fig. 3 Prenatal LPS exposure leads to vasoconstrictor dysfunction in both the thoracic aorta and superior mesenteric artery (SMA) of adult F2 offspring. a-d Vascular reactivity of both the thoracic aorta (a and $\mathbf{c}$ ) and SMA (b and d) rings from 16-week-old F2 offspring. PEinduced cumulative concentration-response curves $(\mathbf{a}, \mathbf{b})$ and ACh-induced relaxation-response curves (c, $\mathbf{d})$ were measured in endotheliaintact thoracic aorta and SMA rings from 16-week-old F2 offspring. Concentration-response curves are expressed as the percentage of contractions caused by $\mathrm{KCl}(60 \mathrm{mM})$, and relaxation-response curves are expressed as the percentage of the maximal PE-induced contractions. Data are presented as means \pm SEM. $n=8$ from 4 litters for each group for a-d. ${ }^{*} P<0.01$ denotes the statistical comparison between two marked groups (two-way ANOVA followed Sidak's multiple comparisons test for a-d). The $\mathrm{p} \mathrm{D}_{2}\left(-\log \mathrm{EC}_{50}\right)$ values were calculated for each group and shown on the top left of each panel.

significance (Fig. 5a). BSP data revealed that the ratio of methylated DNA within the promoter region of Sos1, Sos2, Gng10, and Prkacb was statistically reduced in thoracic aortas of F2 LPS-P offspring as compared to those in F2 control offspring (Fig. 5b and Supplementary Fig. S6).

The most important evidence supporting multi-generational inheritance came from the cytosine methylation patterns of $\mathrm{G} \beta \mathrm{Y}$ signaling pathway in the sperm of F1 offspring. Highly pure (>99\%) sperms isolated from the caudal epididymis of males from both F1 control and F1 LPS-P offspring were isolated for BSP validation of the ratio of methylated DNA within the promoter region of genes clustered in G $\beta \gamma$ signaling. The data demonstrated that the cytosine methylation patterns of Sos1, Sos2, Gng10, and Prkacb in the sperm of PIE F1 LPS-P offspring were similar to those found in the thoracic aorta of F2 LPS-P offspring (Fig. 5c and Supplementary Fig. S7). These data suggested that transgenerational inherited reduced frequency of DNA methylation in the promoter region of genes clustered in $G \beta \gamma$ signaling might play a critical role in the development of multi-generational inherited hypertension.

Activation of PI3K-Akt signaling, downstream of G $\beta Y$ signaling, is implicated in the enhanced vasoconstrictor response in F2 LPS-P offspring

$\mathrm{G} \beta \gamma$ signaling pathway belongs to $\mathrm{G}$ protein signal transduction system that plays critical role in participating hypertension development $[33,34]$. As reported, PI3K $\beta$ could be activated by direct interaction of $\mathrm{G} \beta \gamma$ with $\mathrm{p} 110 \beta[35,36]$, a critical substrate for $\mathrm{PI} 3 \mathrm{~K}$ activation. Activation of PI3K-Akt signaling plays critical roles in the regulation of VSMC proliferation and vascular tone response (Fig. 6a) [37]. Overactivation of PI3K-Akt signaling is usually associated with vascular remodeling and pathological changes [32]. Therefore, we next determined the activity of the critical downstream effector molecules of PI3K-Akt signaling, such as phosphorylated Akt at Ser473 site $\left(\mathrm{p}-\mathrm{Akt}^{\text {Ser473 }}\right)$ and phosphorylated $\mathrm{S} 6$ at Ser235/236 site (p-S6 $\left.{ }^{\text {Ser235/236 }}\right)$, which were also the distinctive substrates for mTORC1 and $\mathrm{mTORC2}$, respectively [38]. The immunoblotting data showed that there was significant increased phosphorylation level of Akt and S6, but not the total protein level of Akt and S6, in thoracic aortas of F2 LPS-P offspring, as compared to those in F2 control offspring (Fig. 6b). This suggested that the activity of PI3K-Akt signaling was enhanced in PIE F2 offspring.

To further assess the role of PI3K-Akt activation in the enhanced arterial contractility of the thoracic aorta of F2 LPS-P offspring, thoracic aortic rings from F2 LPS-P and control rats were isolated and vascular reactivity was tested in the presence of PI3K inhibitor, LY294002 [21]. LY294002 treatment significantly reversed the enhanced PE-induced contractile responses in the thoracic aorta of F2 LPS-P offspring, which even showed no statistical significance with that of F2 control offspring treated with LY294002 (Fig. 6c). In addition, there was no difference of $\mathrm{ACh}$-induced vasodilation of thoracic aortas between both F2 control and F2 LPS-P offspring with LY294002 treatment (Fig. 6d). These data demonstrated that PI3KAkt signaling was critical for the enhanced PE-induced contractile responses of thoracic aortas in F2 PIE offspring.

\section{DISCUSSION}

Most of common genetic variants discovered by genomic studies affecting blood pressure account for less than $4 \%$ of the betweenperson variation in trait [24], indicating that environmental factors are also closely relevant in influencing the inheritance of disease risk [10]. Transgenerational epigenetic inheritance refers to that the transmission of a specific phenotype from parent to the next generation via epigenetic marks in sperm [39]. Our findings 

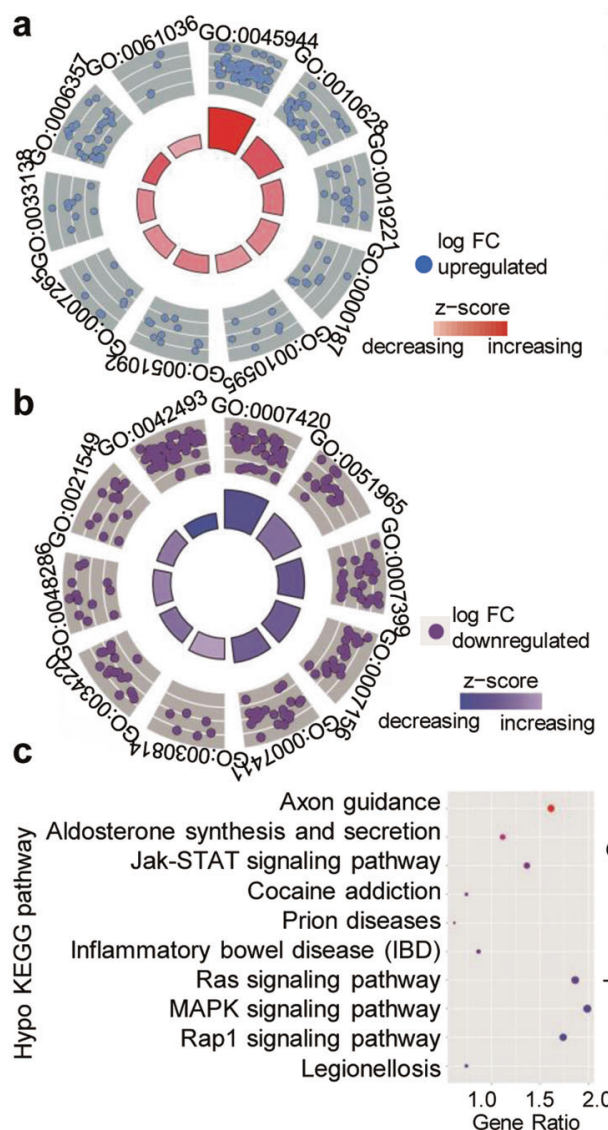

\section{ID Description}

GO:0045944 positive regulation of transcription from RNA polymerase II promoter

GO:0010628 positive regulation of gene expression

GO:0019221 cytokine-mediated signaling pathway

GO:0000187 activation of MAPK activity

GO:0010595 positive regulation of endothelial cell migration

GO:0051092 positive regulation of NF-kappaB transcription factor activity

GO:0007265 Ras protein signal transduction

GO:0033138 positive regulation of peptidyl-serine phosphorylation

GO:0006357 regulation of transcription from RNA polymerase II promoter

GO:0061036 positive regulation of cartilage development

ID Description

GO:0007420 brain development

GO:0051965 positive regulation of synapse assembly

GO:0007399 nervous system development

GO:0007156 homophilic cell adhesion via plasma membrane adhesion

GO:0007411 axon guidance

GO:0030814 regulation of cAMP metabolic process

GO:0034220 ion transmembrane transport

GO:0048286 lung alveolus development

GO:0021549 cerebellum development

GO:0042493 response to drug

GO:0042

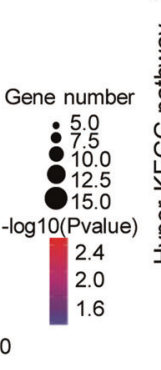

Proteoglycans in cancer Nicotine addiction cAMP signaling pathway Neuroactive ligand-receptor interaction
$\stackrel{\mathbb{N}}{\mathrm{N}}$ Transcriptional misregulation in cancer O) Phosphatidylinositol signaling system TGF-beta signaling pathway Glycosaminoglycan biosynthesis heparan sulfate / heparin Endocytosis Axon guidance

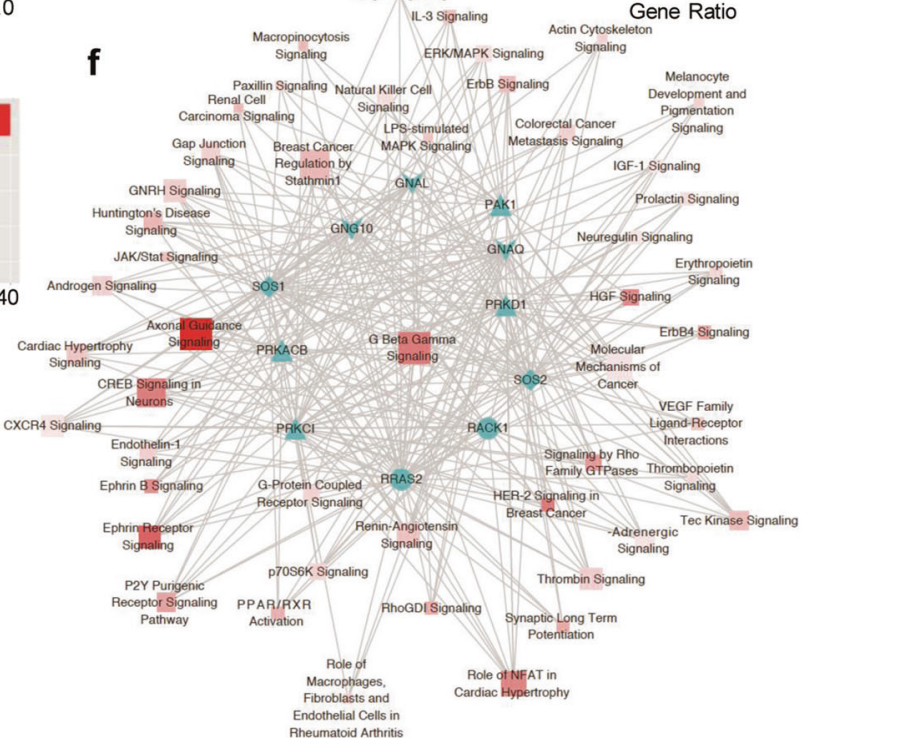

Fig. 4 Characteristic of genomic differentially methylated regions (DMRs) in the thoracic aorta of F2 LPS-P offspring. a, b Significantly enriched Gene Ontology (GO) terms in the biologic process (BP) associated with hypomethylated DMRs (a) and hypermethylated DMRs (b) identified in aortic MeDIP-Seq data of F2 LPS-P offspring relative to control offspring. Each circle represents one gene (left) and the description of each GO item are shown (right). c, d Canonical pathway enrichment of Kyoto Encyclopedia of Genes and Genomes (KEGG) terms from David of hypomethylated DMRs (c) and hypermethylated DMRs (d). The color shade and size of each circle reflect -log $(P$ value) and the number of genes enriched in each KEGG pathway, respectively. $n=4$ from 4 separate litters for each group for a-d. e, $\mathbf{f}$ DMRs functional analysis by Ingenuity pathway analysis (IPA). Top five enriched IPA canonical pathways (e) and the most significantly enriched IPA interaction networks (f) of hypomethylated DMRs identified in aortic MeDIP-Seq data of F2 LPS-P offspring relative to control offspring. The color shade of bar reflects - log $(P$ value) and the horizontal axe represents the number of genes enriched in each canonical pathway, respectively. Red indicates the enriched canonical pathways; blue indicates the nearest genes associated with DMRs identified in aortic MeDIP-Seq data of F2 LPS-P offspring relative to control offspring (f). 


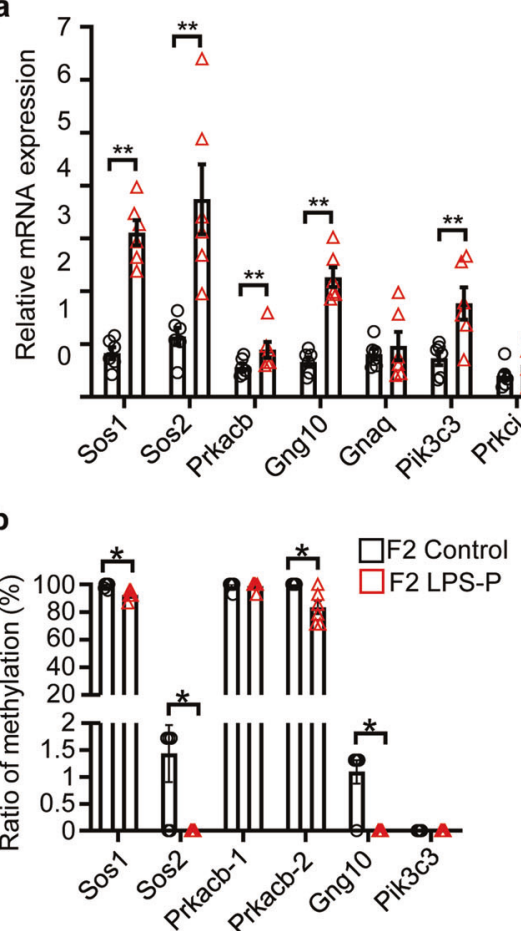

$\square \mathrm{F} 2$ Control F2 LPS-P

Fig. 5 Hypomethylated DMRs within the promoter region along with downregulated mRNA expression of genes clustered in G $\beta \gamma$ signaling exists in both the F2 thoracic aorta and F1 sperm of PIE LPS-P offspring. a Validation of the mRNA expression by qPCR in genes clustered in G $\beta \gamma$ signaling pathway in the thoracic aorta of F2 LPS-P offspring relative to control offspring. Each dot represents one rat. b, $\mathbf{c}$ Validation of DMRs in genes clustered in G $\beta \gamma$ signaling pathway identified in aortic MeDIP-Seq data by bisulfite sequencing PCR (BSP) in both the thoracic aorta of F2 offspring (b) and in the sperm of F1 offspring (c). BSP results were expressed as a percentage of DNA methylation in each sequence, detailed in Supplementary Figs. S4 and S5 for $\mathbf{a}$ and $\mathbf{b}$, respectively.

showed that PIE, irrespective of the kind of inflammation stimulants and the duration in the second trimester, could induce a multi-generation heredity of hypertension, identifying an additional and influential pathway that contributes to the continuous rising incidence of hypertension.

The modification of DNA methylation can arise from early embryos to old age throughout life; however, the most important and extensive modifications occur during embryonic, fetal, and early postnatal life [40]. It could be envisioned that abnormal DNA methylome, caused by PIE at the critical time window for the development of fetal cardiovascular system [41], is a significant event responsible for the multi-generation heredity of hypertension. Notably, our data showed that genes with hypomethylated DMRs were mainly enriched in pathways associated with vascular remodeling and the pathogenesis of hypertension, especially $\mathrm{G} \beta \gamma$ signaling pathways in the core of IPA interaction networks. In rat model of maternal low protein diet, previous study found a significantly undermethylated proximal promoter of the Agtr1b gene, along with upregulated mRNA and protein expression of Agtr $1 b$, in the adrenal of F1 rat offspring [42]. The above evidence suggested that undermethylated DNA methylation might play critical roles in the fetal programming of multi-generational inherited hypertension.

Interestingly, our further experimental studies confirmed the close association of DNA hypomethylation in the promoter region and higher transcription of $G \beta \gamma$ signaling genes in both the sperm of F1 offspring and the thoracic aorta of F2 PIE LPS-P descendants, accompanied by overactivation of downstream PI3K-Akt signaling. Application of $\mathrm{PI} 3 \mathrm{~K}$ inhibitor in the test of vascular reactivity in the thoracic aorta further confirmed the role of enhanced GBY-PI3K-Akt axis in participating the enhanced arterial contractility of thoracic aorta from F2 LPS-P offspring. Our preliminary studies in F1 PIE offspring also showed a similar overactivation of PI3K-Akt signaling in vascular tissue (unpublished data). Previous study has revealed that the activation of PI3K-Akt signaling enhances the spontaneous and agonist-induced contraction in aorta of deoxycorticosterone acetatesalt hypertensive rats [43]. PI3K-Akt signaling has also been reported to mediate the sympathetic and arterial pressure effects of leptin [44]. Therefore, these data demonstrated that PIE-induced multi-generational inherited hypomethylation of genes clustered in $\mathrm{G} \beta \gamma$ signaling, via activation of PI3K-Akt signaling, played a critical role in the development of hypertension in PIE descendants. Of note, we could not still exclude other potential fraction of DNA methylation locus that contribute to the development of F2 hypertension, as we do not carry out a rescue experiment of G $\beta \gamma$ signaling in our study. We could not also exclude the factors that is contributed by PIE female offspring, because we only used the male offspring in this current study,

PIE could be caused not only by "hot" inflammation after pathogens infections (virus, bacterial, and parasites infection), but also by "cold" inflammation in several chronic inflammatory diseases $[3,45]$. It is of great challenge to avoid infection-induced inflammation or chronic inflammatory states during pregnancy. Therefore, exploration of the potential mechanisms related to the altered DNA methylation with tissue specificity in PIE progenies from the aspect of maternal-fetal interface or adolescent F1 offspring would be of immense research benefit. By doing this, it will provide potential specific diagnostic biomarkers and universal therapeutic strategies for the prevention of PIE-programmed transgenerational hypertension.

Conclusively, we have revealed that PIE induces a multigenerational effect on the development of hypertension, which is due to the altered DNA methylome in F2 vascular tissue. In particular, PIE-induced DNA hypomethylation of the G $\beta Y$ signaling pathway in both the F1 sperm and the thoracic aorta of F2 offspring may be responsible for the vascular remodeling and dysregulated vascular tone via affecting PI3K/Akt signaling in PIE F2 offspring. Our findings broaden the current knowledge regarding the multi-generation effect 
a
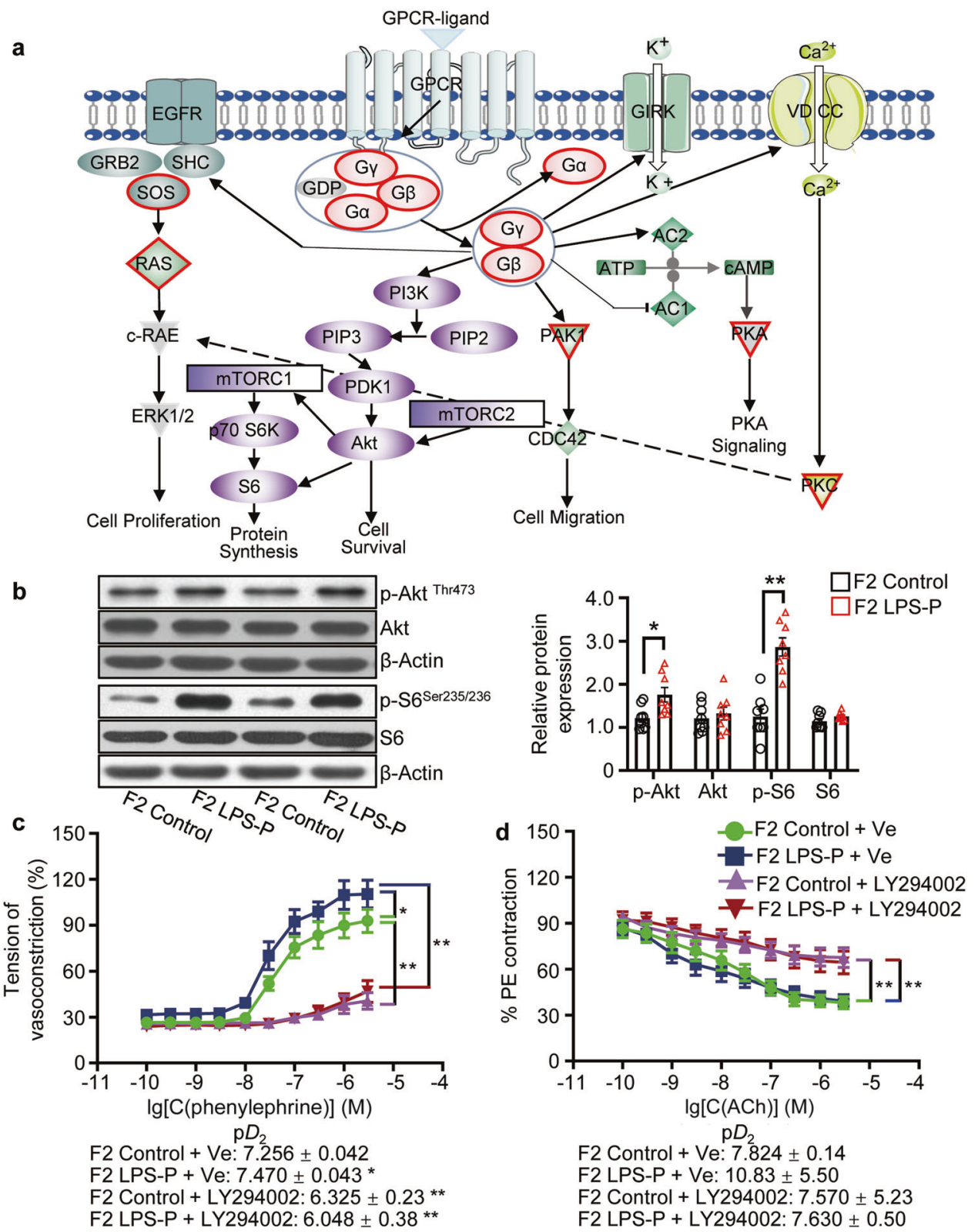

Fig. 6 Activation of PI3K-Akt signaling is implicated in the enhanced vasoconstrictor response in F2 LPS-P offspring. a Illustration of G $\beta \gamma$ Signaling pathway. Genes marked with red border own hypomethylated DMRs within its DNA sequence identified in aortic MeDIP-Seq data of F2 LPS-P offspring relative to control offspring. Genes marked with purple border belong to PI3K-Akt signaling. b Protein levels of phosphorylated (p)-Akt ${ }^{\text {ser473}}, \mathrm{p}-\mathrm{S} 6^{\text {Ser235/236 }}, \mathrm{Akt}$, and S6 in thoracic aortas of F2 offspring at the age of 16 weeks were determined by immunoblotting. $\beta$-actin was taken as internal control. Representative plots of two from eight offspring in each group (left) and statistical data of relative densitometry, normalized by $\beta$-actin (right), are shown. Data are presented as means \pm SEM. ${ }^{*} P<0.05$ and ${ }^{* *} P<0.01$ denote the statistical comparison between the two marked groups (unpaired Student's $t$ test). c, d Effect of PI3K inhibitor LY294002 on the vascular reactivity of aortic rings from 16-week-old F2 offspring. PE-induced cumulative concentration-response curves (c) and Ach-induced relaxation-response curves (d) were measured in endothelia-intact aortic rings after 30 min treatment with LY294002 $(20 \mu \mathrm{M})$ or vehicle control, as described in Fig. 3. Data are presented as means \pm SEM. $n=8$ from 4 litters for each group. ${ }^{*} P<0.05$ and ${ }^{* *} P<0.01$ denote the statistical comparison between the two marked groups (two-way ANOVA followed Sidak's multiple comparisons test for $\mathbf{c}$ and d). The pD2 $\left(-\log \mathrm{EC}_{50}\right)$ values were calculated for each group and shown on the bottom of each panel.

of the cumulative early life environmental factors on the development of hypertension. Further studies are warranted to find similar evidence in human hypertension.

\section{ACKNOWLEDGEMENTS}

This study was supported by National Natural Science Foundation of China (81773742, 81520108029), National Key Research and Development Project
(2019YFA0111200, 2020YFA0113500), and Chongqing Science and Technology Commission of China (cstc2017jcyjBX0058).

\section{AUTHOR CONTRIBUTIONS}

$X G, B B C, Y C D$, and $X H L$ designed the project and analyzed the data. $X G, W N S$, and YCD wrote the manuscript. GRD, WJL, FJW, MM, BHM, YJ, PH, TTY, YFD, LS, and WG performed the animal experiments. $Y Y$ performed the canonical pathways analyzing. XG and GRD performed the qRT-PCR assays and western blots. PY, JHY, YG, and WNS 
gave experiment suggestions or provided experiment materials for this research. All authors read and approved the final manuscript.

\section{ADDITIONAL INFORMATION}

Supplementary information The online version contains supplementary material available at https://doi.org/10.1038/s41401-021-00772-8.

Competing interests: The authors declare no competing interests.

\section{REFERENCES}

1. Coffman TM. Under pressure: the search for the essential mechanisms of hypertension. Nat Med. 2011;17:1402-9.

2. Niiranen TJ, McCabe EL, Larson MG, Henglin M, Lakdawala NK, Vasan RS, et al. Risk for hypertension crosses generations in the community: a multi-generational cohort study. Eur Heart J. 2017;38:2300-8.

3. Deng Y, Song L, Nie X, Shou W, Li X. Prenatal inflammation exposureprogrammed cardiovascular diseases and potential prevention. Pharmacol Ther. 2018;190:159-72.

4. Estes ML, McAllister AK. Maternal immune activation: implications for neuropsychiatric disorders. Science. 2016;353:772-7.

5. Mazumder B, Almond D, Park K, Crimmins EM, Finch CE. Lingering prenatal effects of the 1918 influenza pandemic on cardiovascular disease. J Dev Orig Health Dis. 2010;1:26-34.

6. Cocoros NM, Lash TL, Ozonoff A, Norgaard M, DeMaria A Jr., Andreasen V, et al. Prenatal influenza exposure and cardiovascular events in adulthood. Influenza Other Respir Viruses. 2014;8:83-90.

7. Liguori A, D'Armiento FP, Palagiano A, Palinski W, Napoli C. Maternal C-reactive protein and developmental programming of atherosclerosis. Am J Obstet Gynecol. 2008;198:281 e1-5.

8. Tanacan A, Yazihan N, Erol SA, Anuk AT, Yucel Yetiskin FD, Biriken D, et al. The impact of COVID-19 infection on the cytokine profile of pregnant women: a prospective case-control study. Cytokine. 2021;140:155431.

9. Mendis S, Armstrong T, Bettcher D, Branca F, Lauer J, Mace C, et al. Global status report on noncommunicable diseases 2014. Report No: Stroke. 2014. https:// www.who.int/publications/i/item/9789241564854.

10. Padmanabhan $S$, Joe B. Towards precision medicine for hypertension: a review of genomic, epigenomic, and microbiomic effects on blood pressure in experimental rat models and humans. Physiol Rev. 2017;97:1469-528.

11. Hao XQ, Zhang HG, Yuan ZB, Yang DL, Hao LY, Li XH. Prenatal exposure to lipopolysaccharide alters the intrarenal renin-angiotensin system and renal damage in offspring rats. Hypertens Res. 2010;33:76-82.

12. Wei $\mathrm{YL}$, Li $\mathrm{XH}$, Zhou JZ. Prenatal exposure to lipopolysaccharide results in increases in blood pressure and body weight in rats. Acta Pharmacol Sin. 2007:28:651-6.

13. Liao W, Wei Y, Yu C, Zhou J, Li S, Pang Y, et al. Prenatal exposure to zymosan results in hypertension in adult offspring rats. Clin Exp Pharmacol Physiol. 2008:35:1413-8.

14. Sales VM, Ferguson-Smith AC, Patti ME. Epigenetic mechanisms of transmission of metabolic disease across generations. Cell Metab. 2017;25:559-71.

15. Sharma U, Rando OJ. Metabolic inputs into the epigenome. Cell Metab. 2017;25:544-58

16. Horvath S, Raj K. DNA methylation-based biomarkers and the epigenetic clock theory of ageing. Nat Rev Genet. 2018;19:371-84.

17. Martinez D, Pentinat T, Ribo S, Daviaud C, Bloks VW, Cebria J, et al. In utero undernutrition in male mice programs liver lipid metabolism in the secondgeneration offspring involving altered Lxra DNA methylation. Cell Metab. 2014;19:941-51.

18. Ferguson-Smith AC, Patti ME. You are what your dad ate. Cell Metab. 2011;13:115-7.

19. Deng $Y$, Deng $Y, \mathrm{He} X, \mathrm{Chu} J$, Zhou J, Zhang Q, et al. Prenatal inflammationinduced NF-kappaB dyshomeostasis contributes to renin-angiotensin system over-activity resulting in prenatally programmed hypertension in offspring. Sci Rep. 2016;6:21692.

20. Zhao S, Zhang H, Cao D, Liu Y, Li X. Lipopolysaccharide exposure during pregnancy leads to aortic dysfunction in offspring rats. PLoS One. 2014;9:e102273.

21. Loberg RD, Northcott CA, Watts SW, Brosius FC 3rd. PI3-kinase-induced hyperreactivity in DOCA-salt hypertension is independent of GSK-3 activity. Hypertension. 2003;41:898-902.

22. Kumaki Y, Oda M, Okano M. QUMA: quantification tool for methylation analysis. Nucleic Acids Res. 2008;36:W170-5.

23. Deng Y, Kerdiles $Y$, Chu J, Yuan S, Wang Y, Chen X, et al. Transcription factor foxo1 is a negative regulator of natural killer cell maturation and function. Immunity. 2015;42:457-70.

24. Arnett DK, Claas SA. Omics of blood pressure and hypertension. Circ Res. 2018;122:1409-19.

25. Kelsey G, Stegle O, Reik W. Single-cell epigenomics: recording the past and predicting the future. Science. 2017;358:69-75.

26. Patel RS, Masi S, Taddei S. Understanding the role of genetics in hypertension. Eur Heart J. 2017;38:2309-12.

27. Lacolley P, Regnault V, Segers P, Laurent S. Vascular smooth muscle cells and arterial stiffening: relevance in development, aging, and disease. Physiol Rev. 2017;97:1555-617.

28. Heusch G, Libby P, Gersh B, Yellon D, Bohm M, Lopaschuk G, et al. Cardiovascular remodelling in coronary artery disease and heart failure. Lancet. 2014;383:1933-43.

29. Kato N, Loh M, Takeuchi F, Verweij N, Wang X, Zhang W, et al. Trans-ancestry genome-wide association study identifies 12 genetic loci influencing blood pressure and implicates a role for DNA methylation. Nat Genet. 2015;47:1282-93.

30. Richard MA, Huan T, Ligthart S, Gondalia R, Jhun MA, Brody JA, et al. DNA methylation analysis identifies loci for blood pressure regulation. Am J Hum Genet. 2017;101:888-902.

31. Heard E, Martienssen RA. Transgenerational epigenetic inheritance: myths and mechanisms. Cell. 2014;157:95-109.

32. Hopkins PN. Molecular biology of atherosclerosis. Physiol Rev. 2013;93:1317-542.

33. Belmonte SL, Blaxall BC. G protein coupled receptor kinases as therapeutic targets in cardiovascular disease. Circ Res. 2011;109:309-19.

34. Brinks $H L$, Eckhart AD. Regulation of GPCR signaling in hypertension. Biochim Biophys Acta. 2010;1802:1268-75.

35. Lopez-Ilasaca M, Crespo P, Pellici PG, Gutkind JS, Wetzker R. Linkage of G proteincoupled receptors to the MAPK signaling pathway through PI 3-kinase gamma. Science. 1997;275:394-7.

36. Jo SH, Leblais V, Wang PH, Crow MT, Xiao RP. Phosphatidylinositol 3-kinase functionally compartmentalizes the concurrent $\mathrm{G}(\mathrm{s})$ signaling during beta2adrenergic stimulation. Circ Res. 2002;91:46-53.

37. Sata $M$, Nagai R. Phosphatidylinositol 3-kinase: a key regulator of vascular tone? Circ Res. 2002;91:273-5.

38. Saxton RA, Sabatini DM. mTOR signaling in growth, metabolism, and disease. Cell. 2017;168:960-76.

39. Daxinger $\mathrm{L}$, Whitelaw $\mathrm{E}$. Understanding transgenerational epigenetic inheritance via the gametes in mammals. Nat Rev Genet. 2012;13:153-62.

40. Lane M, Robker RL, Robertson SA. Parenting from before conception. Science. 2014;345:756-60

41. Park C, Kim TM, Malik AB. Transcriptional regulation of endothelial cell and vascular development. Circ Res. 2013;112:1380-400.

42. Bogdarina I, Welham S, King PJ, Burns SP, Clark AJ. Epigenetic modification of the renin-angiotensin system in the fetal programming of hypertension. Circ Res. 2007;100:520-6.

43. Northcott CA, Poy MN, Najjar SM, Watts SW. Phosphoinositide 3-kinase mediates enhanced spontaneous and agonist-induced contraction in aorta of deoxycorticosterone acetate-salt hypertensive rats. Circ Res. 2002;91:360-9.

44. Harlan SM, Rahmouni K. PI3K signaling: a key pathway in the control of sympathetic traffic and arterial pressure by leptin. Mol Metab. 2013;2:69-73.

45. Calay ES, Hotamisligil GS. Turning off the inflammatory, but not the metabolic, flames. Nat Med. 2013;19:265-7. 